\title{
Earth's albedo time series reveals low radiative energy input in December 2020
}

\section{Antti Penttilä ( $\nabla$ antti.i.penttila@helsinki.fi )}

University of Helsinki https://orcid.org/0000-0001-7403-1721

\section{Karri Muinonen}

University of Helsinki https://orcid.org/0000-0001-8058-2642

Olli Ihalainen

\author{
VTT
}

\section{Elizaveta Uvarova}

University of Helsinki

\section{Mikko Vuori}

University of Helsinki

Guanglang Xu

University of Helsinki

Jyri Näränen

Finnish Geospatial Research Institute FGI

\section{Olli Wilkman}

FGI

\section{Jouni Peltoniemi}

Finnish Geospatial Research Institute FGI https://orcid.org/0000-0002-4701-128X

\section{Maria Gritsevich}

Finnish Geospatial Research Institute FGI

\section{Heikki Järvinen}

University of Helsinki

\section{Alexander Marshak \\ NASA Goddard Space Flight Center}

Article

Keywords: spherical albedo, Earth Polychromatic Imaging Camera, seasonal variation

Posted Date: July 16th, 2021

DOI: https://doi.org/10.21203/rs.3.rs-677927/v1 
License: (c) (i) This work is licensed under a Creative Commons Attribution 4.0 International License. Read Full License 
1 Earth's albedo time series reveals low radiative energy in put in December

22020

3 A. Penttilä ${ }^{1 *}$, K. Muinonen ${ }^{1,2}$, O. Ihalainen ${ }^{1,3}$, E. Uvarova ${ }^{1}$, M. Vuori ${ }^{1}$, G. Xu ${ }^{1,5}$, J. Näränen ${ }^{2}$, 4 O. Wilkman ${ }^{2}$, J. Peltoniemi², M. Gritsevich ${ }^{2,6}$, H. Järvinen ${ }^{4}$, A. Marshak ${ }^{7}$

${ }^{1}$ Department of Physics, University of Helsinki, Finland ${ }^{2}$ Finnish Geospatial Research Institute FGI, National Land Survey of Finland, Finland ${ }^{3}$ VTT Technical Research Centre of Finland Ltd., Finland ${ }^{4}$ Institute for Atmospheric and Earth System Research, University of Helsinki, Finland ${ }^{5}$ Institute of Meteorology and Climate Research, Karlsruhe Institute of Technology, Germany ${ }^{6}$ Institute of Physics and Technology, Ural Federal University, Ekaterinburg, Russia ${ }^{7}$ NASA Goddard Space Flight Center, MD, USA

Abstract

The Earth's spherical albedo describes the ratio of light reflected from the Earth to that incident from the Sun, an important input variable for the Earth's radiation balance. The spherical albedo has been previously estimated from satellites in low-Earth orbits, and from light reflected from the Moon. However, neither of these methods can produce continuous time series of the entire planet. We developed a global method to derive the Earth's spherical albedo using the images from the Earth Polychromatic Imaging Camera (EPIC) on board NOAA's Deep Space Climate Observatory (DSCOVR). The satellite is located in the Lagrange 1 point between the Earth and the Sun and observes the complete illuminated part of the Earth at once. The method allows us to provide continuously updated spherical albedo time series data starting from 2015. This time series shows a systematic seasonal variation with the mean annual albedo estimated as $0.295 \pm 0.008$ and an exceptional albedo maximum in 2020 , attributed to unu sually abundant cloudiness over the Southern Oceans.

Solar radiation is the primary energy source of the Earth and largely determines Earth's climate. The proportion of the incoming solar radiation reflected back to space by the Earth is described by the spherical albedo. It depends on the reflective properties of the Earth and thus it is affected by the proportion of the highly reflective areas relative to darker areas. For example, the melting of the Antarctic and Greenland ice sheets results in increased absorption and decreased albedo. Hence, the Earth's spherical albedo is a major factor behind the global weather and climate processes. However, up to date there has not been a global, day-to-day estimate of the spherical albedo.

In the first half of the 20th century, estimates of the spherical albedo were based on an indirect method of observing the Earth-lit Moon ${ }^{1}$. These so-called earthshine observations depend on correct estimations of the Moon's light-scattering properties, and thus they can be inaccurate and vary considerably. The earliest satellite measurement of the spherical albedo was made in 1959 by the Explorer 7 satellite and its value has remained approximately 0.3 ever since ${ }^{2}$. Since 1997 the albedo 
five satellites and seven CERES instruments ${ }^{3}$. As of 2017, only five instruments are operational. As it takes numerous hours for the CERES to scan the entire Earth while the cloud cover of the Earth evolves in a matter of minutes, the spherical albedo evaluation method by the CERES instruments produces noticeable uncertainties in the measured albedo value ${ }^{2,3}$.

To measure the spherical albedo directly one needs to simultaneously detect radiation reflected by the Earth from all directions, which renders such measurements impossible. To circumvent this, we use the Earth Polychromatic Imaging Camera (EPIC) on board the Deep Space Climate Observatory (DSCOVR) spacecraft combined with angular distribution models provided by the CERES based on many years of dedicated measurements. The DSCOVR is a spacecraft orbiting in the Lagrange point 1 around 1.5 million kilometers from the Earth, which allows the EPIC to always view practically the entire sunlit hemisphere of the Earth. The DSCOVR was launched in 2015, and the EPIC has been operational ever since apart from one six-month maintenance break in $2019^{4}$.

Data from the instruments allows us to propose an algorithm that automatically translates directional reflectance obtained from the EPIC images into estimated value of short-wave spherical albedo. We have launched a web service that collects the computed spherical albedo of the Earth from the whole operational period and updates the data daily with the latest observations ${ }^{5}$. This is the first global daily time series of the Earth's albedo and it spans already now over seven years in time. This enables us to analyze the pattern of temporal variation in albedo over a year which demonstrates anomalies in albedo behavior. The accurate estimate of the short-wave spherical albedo is important in evaluating the energy balance of the Earth system.

\section{Results}

The EPIC images constitute a time series of the sunlit part of the Earth, including atmosphere, starting from June 2015 and still running. There are usually about 22 images per day during Northern Hemisphere summer, and 13 during winter. Each multispectral image has 10 wavelength channels between 317 and $780 \mathrm{~nm}$. The channels have full-width-at-half-maximum values between 1 and $3 \mathrm{~nm}^{6-9}$.

Each image pixel represents the radiance reflected by the corresponding surface area on the Earth. This radiance is a function of the reflective properties of the surface, and the solar and satellite angles. The DSCOVR satellite is located in the first Lagrange point between the Earth and the Sun, therefore the radiance is observed close to backscattering geometry with satellite and solar angles being almost equal. To convert measured radiances at backscattering into integrated albedo values at top-of-atmosphere (ToA), we apply the angular distribution models (ADMs) provided by the CERES project ${ }^{10,11}$. The ADMs are provided for several surface types and cloud fractions, and we combine these into four types for our analysis: clear and cloudy land and ocean. The reason for this is that the angular resolution of the tabulated ADMs is only 10 degrees, and we want to interpolate with a finer resolution in the backscattering direction and combining similar surface types gives us more robust estimation (see Methods). Additionally, estimating temporary cloud fractions from EPIC images is challenging, a more robust method can be developed for simple clear versus cloudcovered surface estimation. Land or ocean surface classification is available from the International Geosphere-Biosphere Programme, and temporal cloud coverage we estimate from the EPIC images using logistic regression model with input from EPIC channels at 325, 551, and $780 \mathrm{~nm}$. 
The ToA albedo values for each pixel over the Earth's sunlit disk are averaged for albedo of the Earth at each wavelength channel. Finally, the per-wavelength albedos are summed with weights from incident solar spectra at each channel, obtained from National Oceanic and Atmospheric Administration (NOAA) climate data record of solar spectral irradiance ${ }^{12}$. The solar spectrum is taken as constant in this study with only the distance between the Earth and the Sun influencing the total flux level. The channels at 688 and $764 \mathrm{~nm}$ in EPIC images are left out since they are targeted to measure atmospheric $\mathrm{O}_{2}$ at narrow absorption bands. The resulting time series of Earth's daily albedo is shown in Figure 1.

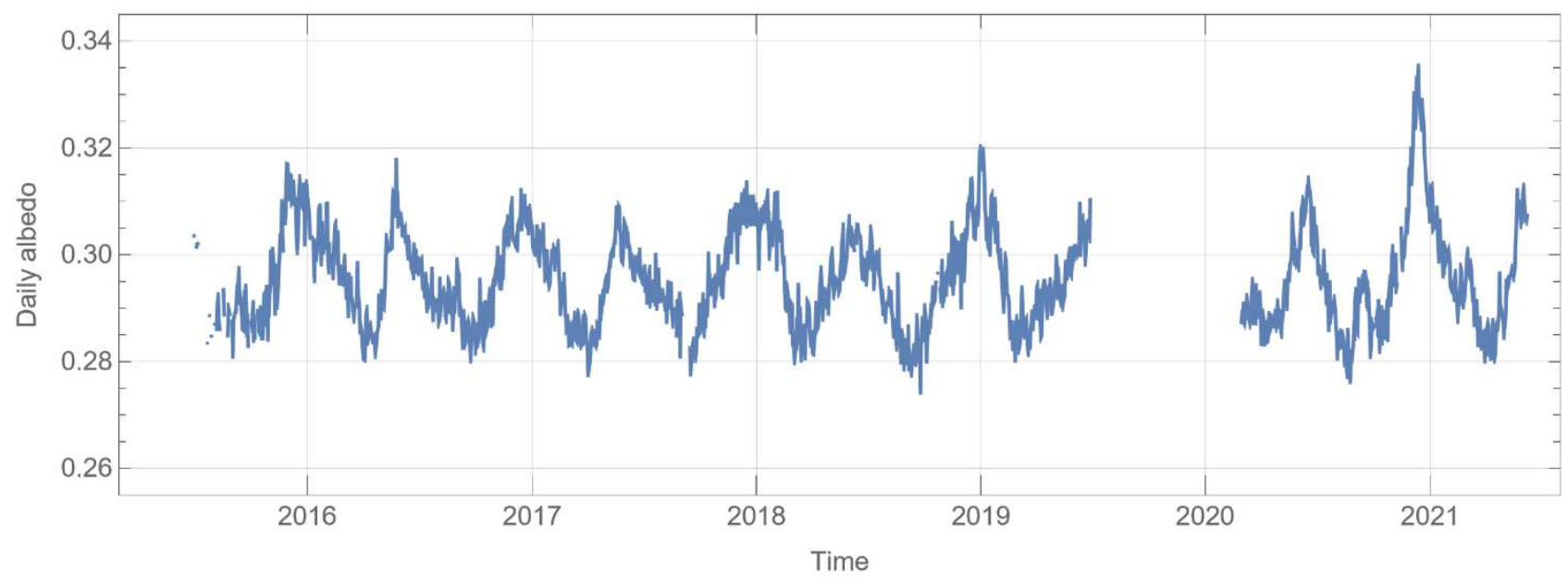

Figure 1: Daily average of Earth's albedo over the whole operational time of the EPIC camera.

The daily averaged time series has been filtered to remove outliers. For some EPIC images, not all the wavelength channels are present. If there is not a proper coverage of usable images over the day, some parts of the Earth are not present, and this would introduce a bias to the mean albedo of that day. Therefore, these days are left out from our time series data. There is a period between late 2019 and early 2020 when the EPIC camera was not operational.

We can derive the mean yearly spherical albedo of the Earth, at visual wavelengths, by grouping the daily time series values by the day in the year, averaging per day, and finally averaging over days in a year. From the currently available data, this value is $29.5 \pm 0.8 \%$. The value agrees well with earlier estimates of 28.6-30.1\% by satellites in low-Earth orbits, see Kandel and Viollier $(2010)^{2}$, and $29.7 \%$ from earthshine measurements done by observing the Moon, see Goode et al. $(2001)^{14}$.

There is an evident annual cycle of the albedo. This can be seen more clearly from Figure 2 where the years have been stacked together. The year 2020 is highlighted with a red color because of the anomaly in the second annual maximum usually present between December and January. In the EPIC data, there are observations of four other Northern Hemisphere winter maxima, but the one in 2020-21 is significantly higher. 


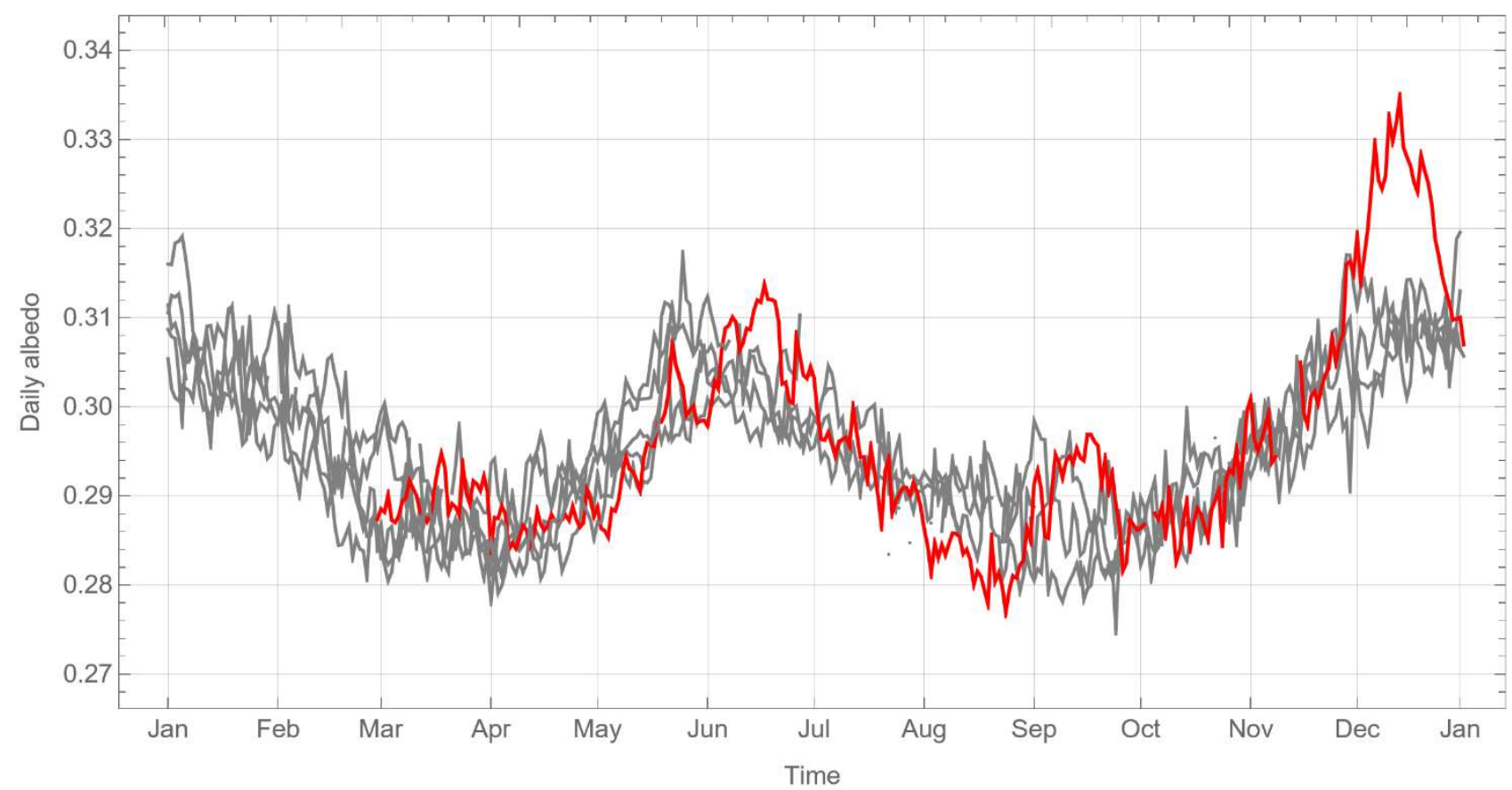

Figure 2: Daily averages of Earth's albedo with all years stacked together. Data from the year 2020 is highlighted with

111 We can verify the anomaly in December 2020 statistically by studying the monthly averages for each year. The combined monthly averages are shown in Figure 3, plotted as variations from the overall average albedo in a box-and-whiskers chart. The one-way analysis-of-variance test of the differences in the mean values over several sets of observations (different years over a month) can find significant ( $p$-value less than $0.1 \%$ ) differences for all months. However, by far the smallest $p$ value, and therefore the largest difference, is for December, formally less than $10^{-29}$.

When conducting $t$-tests for year pairs inside a month, we find that the only year which differs from all the other years when using the Bonferroni-corrected $p$-value limit of $0.001 / m_{i}$, where $m_{i}$ is the number of year data sets on month $i$, is the year 2020 and months August and December. From these analyses we conclude that the largest yearly variations in a month are on December, and that is mainly due to year 2020 . 


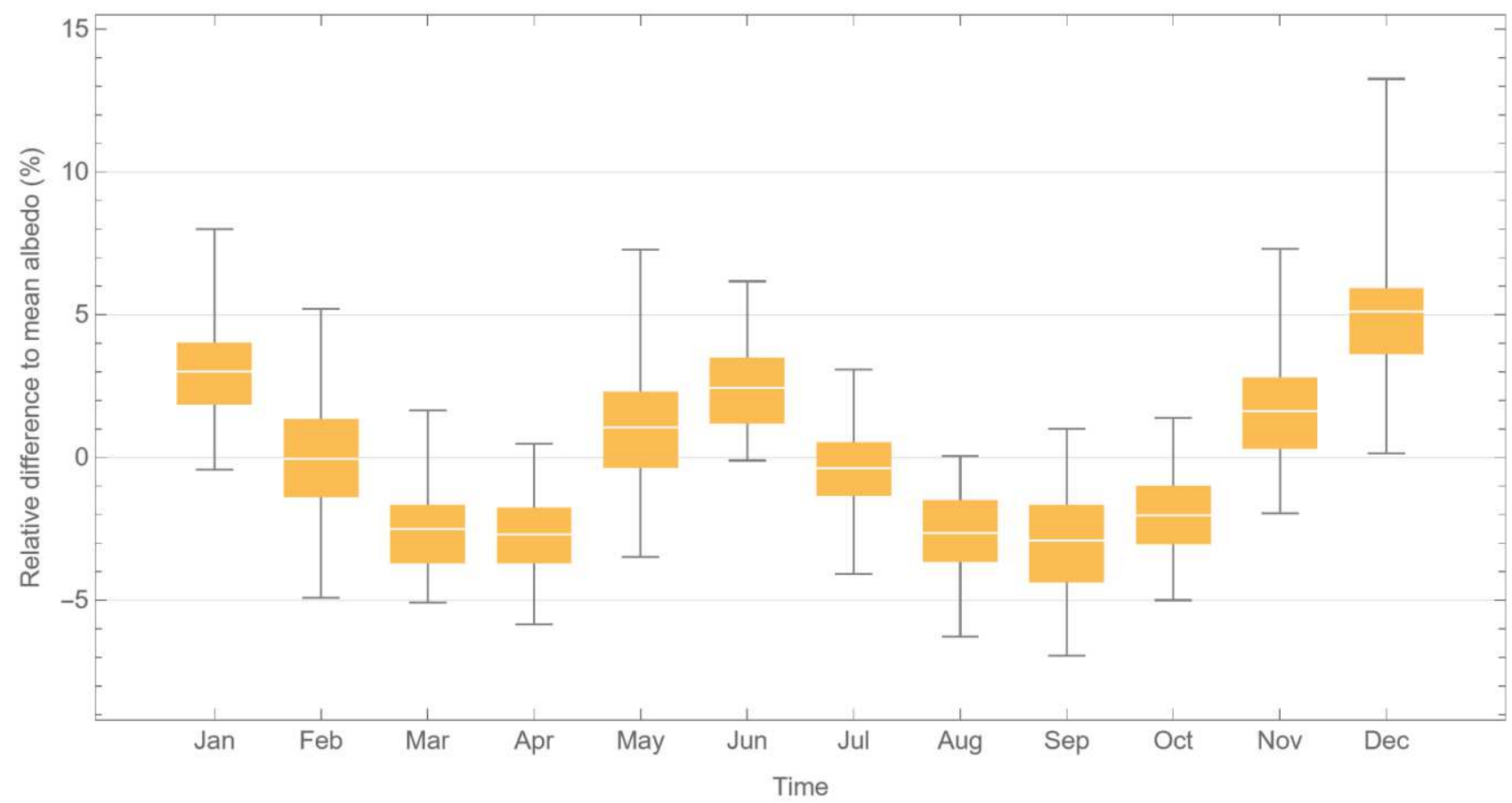

Figure 3: Monthly variation in the daily averages of Earth's spherical albedo. Values are relative differences from the overall mean albedo of 0.295 . The horizontal white line shows the average over the month, the box indicates the range from $1^{\text {st }}$ quarter to $3^{\text {rd }}$ quarter, and the lines the range from minimum to maximum.

We can conclude on the results on temporal variation on Earth's spherical albedo by summarizing that there are two maxima and two minima. The main maximum occurs in December-January during the Southern Hemisphere summer, and the secondary maximum in June during the Northern Hemisphere summer. The minimum values are on March-April, and on September. The coefficient of variation, that is the standard deviation divided by mean, of the daily average values over a year is $2.7 \%$, and the range of minimum-to-maximum deviation of the daily averages from annual average is 0.031 (10.3 percentage points in scale relative to the mean annual albedo). In December 2020, the daily albedo values were up to 0.023 (7.2\%, on Dec 13) larger than the average daily value over all the years.

\section{Discussion}

The annual variation of the Earth's spherical (Bond) albedo is described for the first time in detail. Previous investigations mainly describe the variations on the incident solar flux due to the Earth's elliptic orbit (see Fig. 5 in Kandel and Viollier 2010²), as for us, this effect is considered and properly accounted for. The accurate annual albedo behavior enables the detailed analysis of local geographic and atmospheric effects on the albedo during a year.

The Earth contains regions of low albedo (e.g., cloud-free ocean areas, vegetated land areas) and high albedo (e.g., clouds, ice and snow surfaces, sandy deserts). Diurnal and annual variations in the albedo time series (Figure 1, Figure 3) result from a modulation of these two aspects, depending on the apparent longitude (diurnal variations) and latitude (annual variations). Especially, the cloudcovered ocean areas increase the albedo. The daily cloud-covered ocean fraction, estimated from the EPIC images, has the correlation coefficient value of $86 \%$ with the daily albedo value (see Figure 6). 
149 In annual albedo variations, the main albedo maximum occurs in December when the Antarctic ice

150 sheet, sea ice, and snow cover are visible entirely. At this time, cloud formations of the mid-latitude

151 cyclones over the Southern Ocean are well pronounced. Albedo is further enhanced by the shallow

152 convective cloud cover over the subtropical oceans and the relatively small areas of cloud-free

153 ocean areas visible, on average, at this time of the year.

154 The secondary albedo maximum occurs in June when the Greenland ice sheet and sea ice in the

155 Arctic Ocean are well exposed. Cloud formations of the mid-latitude cyclones of the North Atlantic 156 and Pacific storm tracks are active, although reduced from their winter maxima. Subtropical desert 157 areas are mostly cloud free exposing also these high surface albedo areas. Deep convective clouds 158 of the inter-tropical convergence zone are on the Northern Hemisphere at this time of the year. 159 Again, relatively little cloud-free ocean areas are visible.

160 The main albedo minimum precedes the main maximum in August-September when neither of the 161 Polar regions is visible. At this time of year, there are relatively few shallow convective clouds over 162 the subtropical Northern Atlantic and Pacific, as well as over the Mediterranean. North Atlantic and 163 Pacific storm track activities are near to their minima. Also, vegetation is at its maximum extent in 164 the Sahel region. Monsoon is in active phase over the Indian peninsula and South-East Asia, thus 165 enhancing the albedo. The secondary minimum precedes the secondary maximum in March-April.

166 The solar energy input at the Earth's average distance from the Sun is $1361 \mathrm{~W} / \mathrm{m}^{2}$ for the sunlit 167 disk of the Earth over all wavelengths, and the EPIC filters range from $317 \mathrm{~nm}$ to $780 \mathrm{~nm}$ in 168 wavelength ${ }^{12}$. The portion of the solar in put between these wavelengths is $52.7 \%$. In ultraviolet 169 below $317 \mathrm{~nm}$ there is only $0.5 \%$ of the total energy. We assume that the albedo derived here for 170 the range $317-780 \mathrm{~nm}$ is valid for somewhat longer wavelengths and estimate roughly that our 171 shortwave albedo is valid for at least $60 \%$ of the reflection or absorption of the total solar input. 172 With this estimate, the input energy that is absorbed into the area of sunlit Earth's disk and 173 atmosphere is about $4.9 \mathrm{~W} / \mathrm{m}^{2}$ more during the albedo minimum in September than on average. 174 During the albedo maximum in December, about $8.7 \mathrm{~W} / \mathrm{m}^{2}$ less energy is absorbed.

175 An exceptional albedo occurred on 13 December 2020 and persisted for about a week before and 176 after the peak value. This anomaly coincides with the main annual maximum in December. Our 177 main explanation is a short-lived and exceptional maximum in the amount of shallow convective 178 clouds over the subtropical Indian Ocean and the Pacific east of Australia, see Figure 4. The 179 development of exceptionally wide shallow cloud cover coincides and is favoured by a constellation 180 of several mature mid-latitude cyclones, feeding moist air from the subtropics towards the 181 Antarctic. At the time of the maximum albedo, all relevant large-scale atmosphere-ocean indicators 182 were in close-to-neutral stages: the Madden-Julian oscillation (MJO) phase centre was in the 183 Oceanic continent, but the amplitude was weak; the Indian Ocean Dipole (IOD) was neutral; El 184 Niño - Southern Oscillation (ENSO) was in a weak La Niña phase (El Niño 3.4 index) and 185 returning to neutral. 

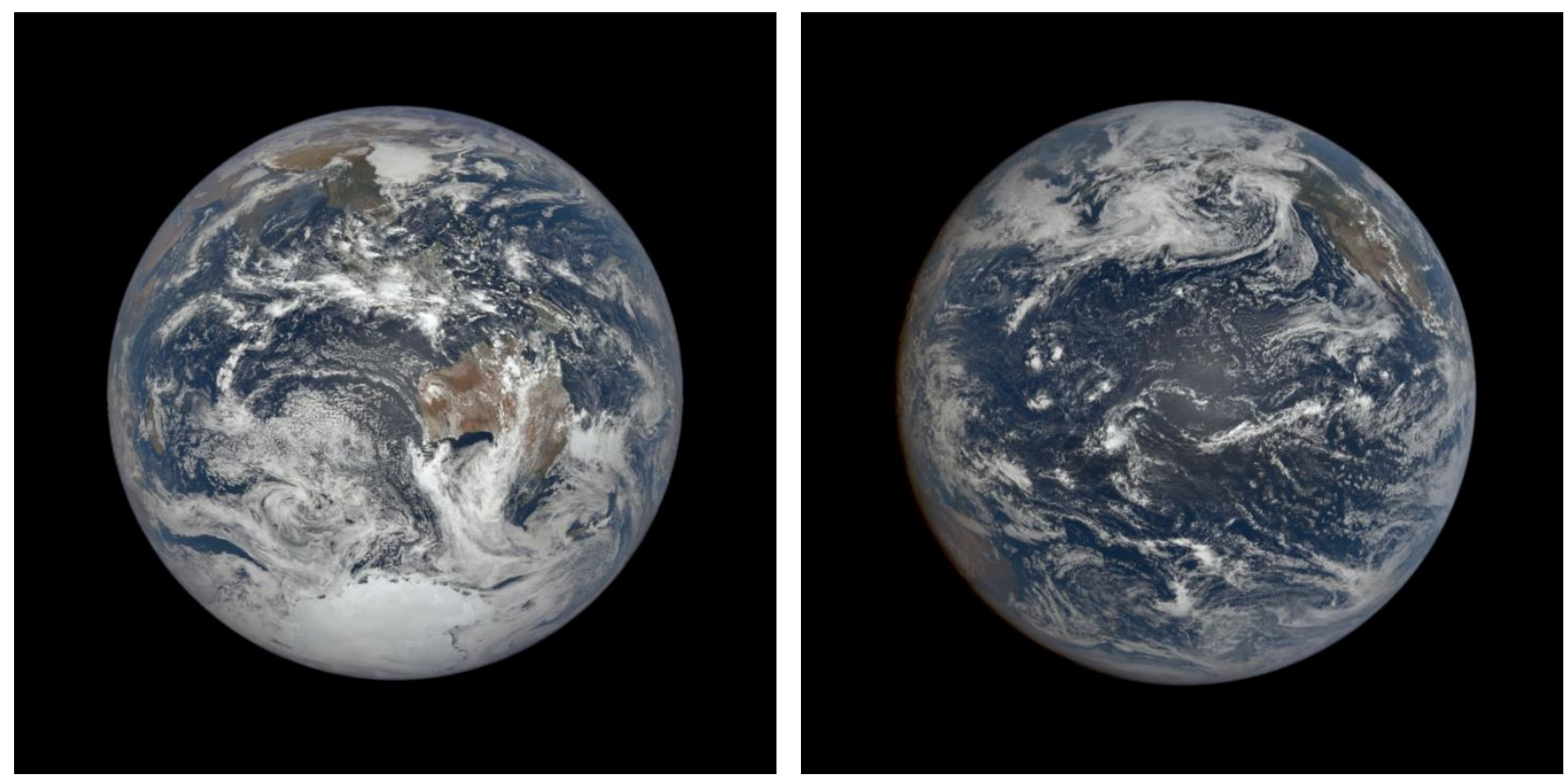

Figure 4: EPIC natural color constructed images, taken at 2020-12-13 at 04:40 UCT (left), and 2020-08-04 at 22:25 UCT (right). The image on the left has one of the largest albedos in 2020 (0.350), and the image on the right has one of the lowest (0.253). Image credits: NASA EPIC team, https://epic.gsfc.nasa.gov/.

In December 2020, the EPIC-derived daily albedo was elevated to 0.334 from its typical December value of 0.312 in 2015-19. Assuming these to represent well the planetary reflectivity, the emission temperature of the Earth would decrease by $2.1 \mathrm{~K}$ through a $7.8 \mathrm{~W} / \mathrm{m}^{2}$ reduction in the absorbed solar radiation. For comparison, the global mean sensible heat flux from the Earth surface to the atmosphere amounts to about $20 \mathrm{~W} / \mathrm{m}^{2}$ and the absorbed solar radiation in the atmosphere to about $80 \mathrm{~W} / \mathrm{m}^{2}{ }^{14}$. It is therefore fair to treat the short-term albedo peak in December 2020 as quite remarkable.

\section{Methods}

\section{Cloud classification in EPIC images}

The algorithm for cloud classification in the EPIC images was trained with a manually prepared data set of spectral radiances together with subjective cloudy/clear label decision. For image data, we collected images from the first week of every month in 2018. We converted the original HDF5 library data for one multi-channel image into PNG image stack of all the wavelength channels. We opened the image stacks in Image ${ }^{15}$, and manually selected areas of either clear or cloud-covered land and ocean surfaces. The spectral radiance values of pixels within these areas were recorded together with the label (1) clear land / (2) clear ocean / (3) cloud-covered surface. Altogether, we produced 338 areas of clear land, 331 areas of clear ocean, and 481 areas of cloud-covered surfaces. Each area contains several tens of pixels, the mean values over the areas were employed in the analysis. The ice sheet covered areas, such as Antarctica, are indistinguishable from cloud-covered surfaces. 
A logistic regression model was separately fitted into data with clear land and cloud-covered surfaces, and data with clear ocean and cloud-covered surfaces. A logistic regression model has a linear function modeling the log-odds of the probability of an event. In our case, the event is that the surface is covered with clouds. With some algebra the model can be written as

$$
p=\frac{1}{1+e^{-\left(\beta_{0}+\beta_{1} x_{1}+\cdots+\beta_{k} x_{k}\right)}}
$$

where $p$ is the probability of cloud-cover, and $\beta_{0}+\beta_{1} x_{1}+\cdots+\beta_{k} x_{k}$ is the linear function with unknown coefficients $\beta_{i}$ and known radiances $x_{i}$ from channels $1, \ldots, k$.

We executed forward-selection stepwise regression to optimize the model with the best value of the Bayesian Information Criterion (BIC) statistics of the model. We ended up with models with a constant coefficient $\beta_{0}$ and three coefficients for the EPIC wavelength channels at 325, 551, and $780 \mathrm{~nm}$. For land vs. clouds model, all the four coefficients were tested significant with $p$-values less than $0.1 \%$. For ocean vs. clouds model, all other but the coefficient for the channel $780 \mathrm{~nm}$ were significant with the same $p$-value limit.

Scattering geometry and interpolation of angular distribution models

We found it realistic to successfully classify pixels of clear or cloudy land or ocean surface from the EPIC images, but not different cloud types (water or ice) or levels of cloud cover that are present in CERES angular distribution models (ADMs). ADMs are also divided into different wind speeds for ocean and into different vegetation types for land. To exploit these, wind speeds on ocean pixels would need to be connected from weather models into our analysis together with up-to-date land cover information. This is a bit cumbersome but could be done. However, we decided to emphasize more the interpolation of the scattering geometry inside the ADM models over having many ocean/surface/cloud subtype ADMs.

The scattering geometry in the EPIC observations is such that the observation is always done close to backscattering. The phase angle between the Sun and the DSCOVR spacecraft, as seen from the Earth, is below $12^{\circ}$ except for few rare cases in years 2020-21. On average, phase angle has been $8.2^{\circ}$. After the break in the DSCOVR operations in 2019 , the minimum phase angle has gradually started to decrease (see Figure 6). It was never below $4^{\circ}$ before 2020, but now the minimum value is $1.8^{\circ}$.

While in terms of phase angle EPIC is observing close to backscattering, the local Sun zenith angle on each Earth pixel varies between $0^{\circ}$ and $90^{\circ}$. The ADM tables, on the other hand, have $10^{\circ}$ bins for all the three angles (Sun zenith, observer zenith, azimuth). So, our backscattering geometry implies that we need ADM table values inside cells where Sun and observer zenith angles are in the same bin and azimuth angle is in the bin $0-10^{\circ}$. Thus, the 3 -dimensional (in angle) ADM tables will reduce for our purposes to 1-dimensional tables that can be parametrized with Sun zenith angle only.

We find the reduced 1-d table binning of $10^{\circ}$ too coarse as it would introduce inaccuracies when applied to pixels having Sun zenith angle anywhere between $0^{\circ}$ and $90^{\circ}$. Therefore, we interpolate the reduced $\mathrm{ADM}$ tables over $0^{\circ}$ and $90^{\circ}$ range and use these interpolated coefficients when converting ToA radiances measured by EPIC into integrated ToA albedos. We find that the reduced 1-d tables show relatively similar behavior for all clear ocean surfaces regardless of the wind speed, for all clear land surfaces, and for all cloud-covered surfaces regardless of the cloud type or 
coverage. Thus, our final set of ADM coefficients come from interpolated data on these three categories, averaged over their subtypes.

The phase angle of EPIC observations varies between $2^{\circ}$ and $12^{\circ}$. After DSCOVR operations break in 2019 the phase angle variation has increases and the minimum phase angle has decreased from previous $4^{\circ}$ to $1.8^{\circ}$. These decreasing phase angles introduce possible source of bias in our albedo estimation. The resolution in the binned data of the ADM models is $10^{\circ}$ in all three angles, indicating that we do not have exact information on how possible backscattering effects (selfshadowing, coherent backscattering) are behaving on small phase angles inside this bin.

Currently, we can only estimate the possible bias resulting from small phase angles. We can do this by computing the deviations of daily albedo values from the average-year albedo values, and by modeling how they are changing as a function of the observation phase angle. From Figure 5 we can see that there is obvious correlation between these. Decreasing phase angles are introducing a positive bias, on average, into the albedo values. A second-degree linear fit has the $p$-values of all the model coefficients significant below $0.1 \%$ level, and a slightly better value $(0.37)$ of adjusted $R^{2}$ measure of the model than with first-degree model (0.36). According to this model, there can be up to 0.01 positive average bias coming from the phase angle effect if phase angle approaches $2^{\circ}$.

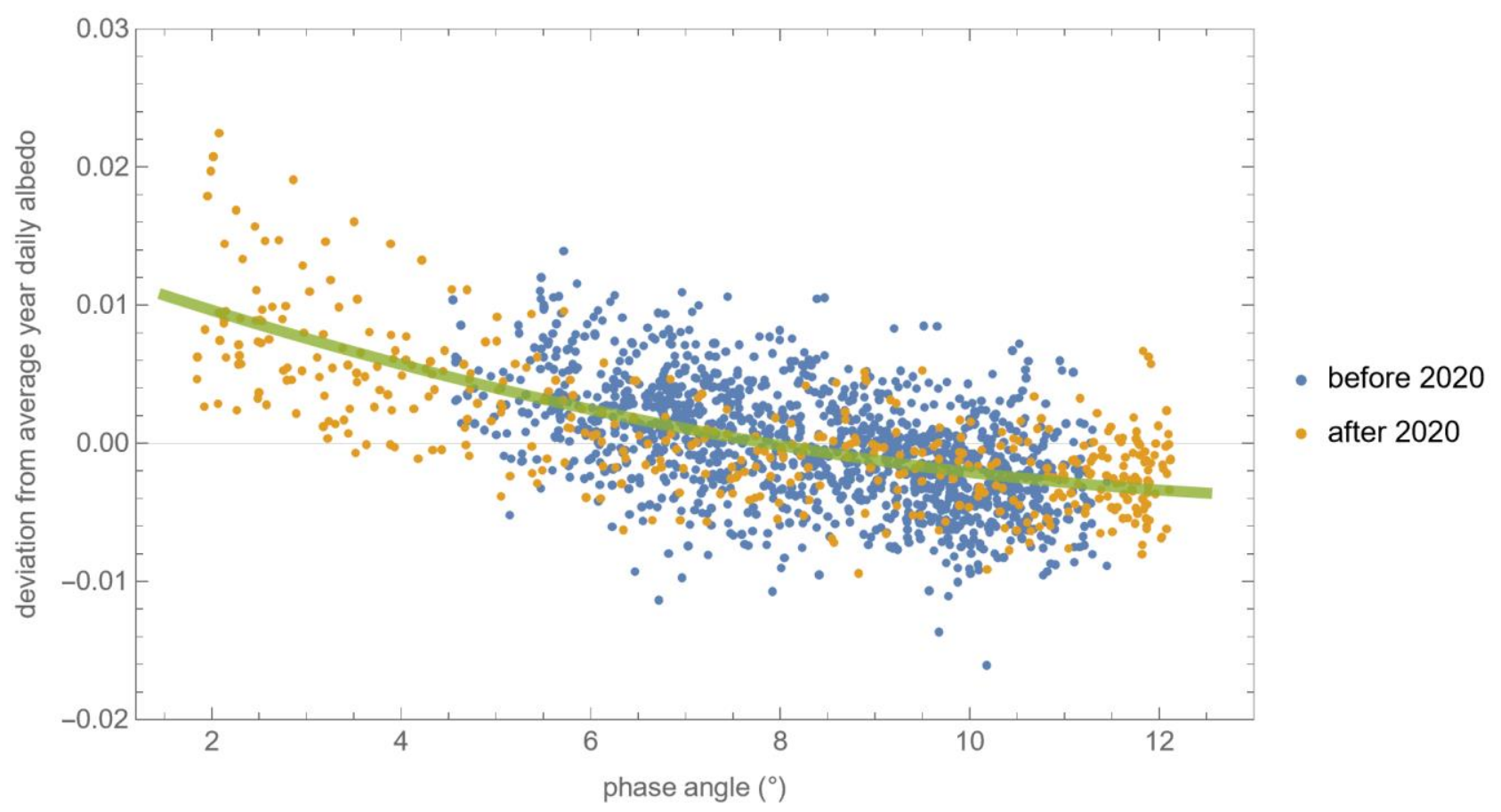

Figure 5: Differences between daily albedo values and corresponding values from average year, as a function of the phase angle of the observation. The solid line shows the 2 nd degree linear fit to data. The data before and after the DSCOVR operations break in 2019-2020 is shown with different colors, but the model is fit on both sets together.

This same bias can also be roughly estimated from the small upturns in daily albedo values in November 2020 and March 2021, see Figure 1 and Figure 6. These upturns are simultaneous with local phase angle minima of about $2^{\circ}$. Graphical estimation of possible excess in albedo values in these upturns also suggests a value of 0.01 at most. 


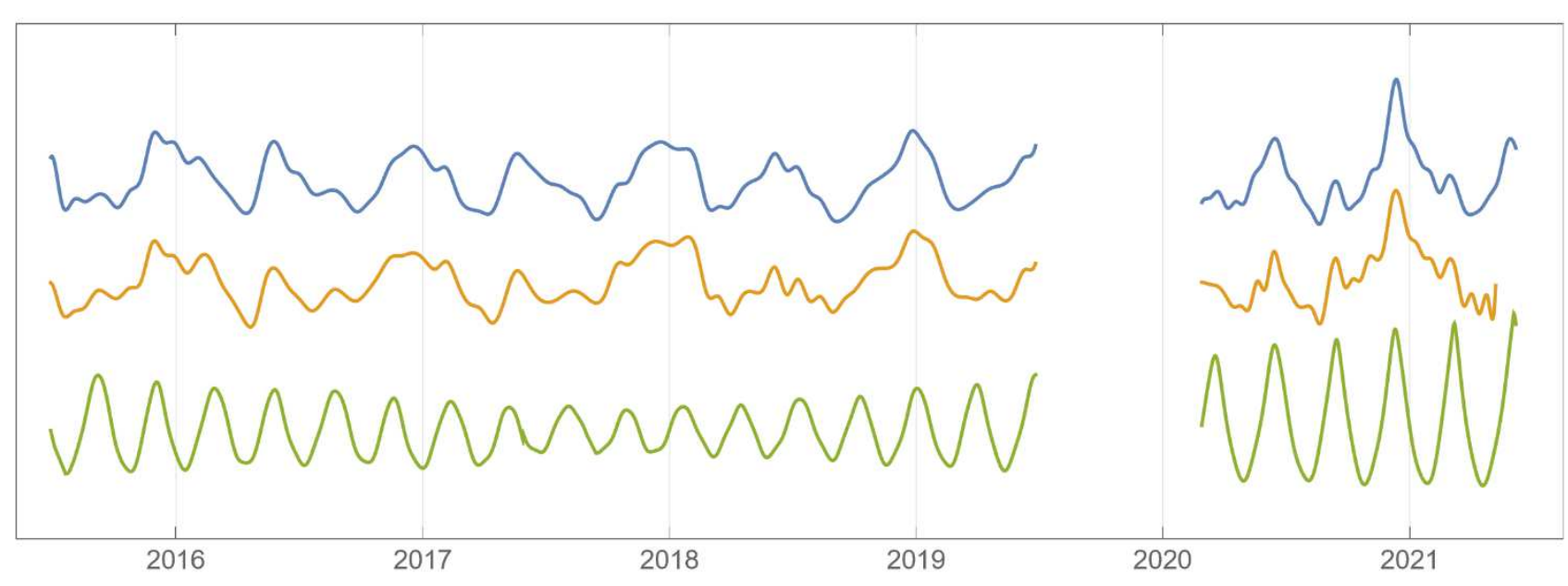

Figure 6: Daily average albedo values (blue lines), daily average fraction of clouds over oceans (orange lines), and scattering angle of EPIC observations (green lines). The scattering angle is the complement of the phase angle $\alpha$, that is, $180^{\circ}-\alpha$. The curves have been shifted in vertical direction and rescaled for improved visualization.

The exceptional albedo values in December 2020 were at most 0.023 larger than the average December value. This exceptional value was received at the time of local phase angle minima of the DSCOVR spacecraft, $2.1^{\circ}$. We conclude that the effect of the phase angle can explain, at maximum, about half of the difference, and that December 2020 is exceptional in our albedo time series even if taking the possible phase angle effect into account.

\section{References}

1 Stephens GL, O'Brien D, Webster PJ, Pilewski P, Kato S, and Li J (2015). The albedo of Earth. Reviews of Geophysics 53(1), 141-163. DOI: 10.1002/2014RG000449.

2 Kandel R and Viollier M (2010). Observation of the Earth's radiation budget from space. Comptes Rendus Geoscience, 342(4), 286-300. DOI: j.crte.2010.01.005.

3 Wielicki BA, Barkstrom BR, Harrison EF, Lee RB, Smith GL, and Cooper JE (1996). Clouds and the Earth's Radiant Energy System (CERES): An Earth Observing System Experiment. Bulletin of the American Meteorological Society 77(5), 853-868. DOI: 10.1175/15200477(1996)077<0853:CATERE>2.0.CO;2.

4 Marshak A, Herman J, Adam S, et al. (2018). Earth observations from DSCOVR EPIC instrument. Bulletin of the American Meteorological Society 99(9), 1829-1850. DOI: 10.1175/BAMS-D-17-0223.1.

5 Earth Spherical Albedo — From DSCOVR/EPIC Satellite Images. Web page, http://albedo.physics.helsinki.fi/.

6 Herman JR, Huang L, McPeters RD, Ziemke J, Cede A, and Blank K (2018). Synoptic ozone, cloud reflectivity, and erythemal irradiance from sunrise to sunset for the whole Earth as viewed by DSCOVR spacecraft from the earth-sun Lagrange-1. Atmospheric Measurement Techniques 11, 1-18.

7 Geogdzhayev I, and Marshak A (2018). Calibration of the DSCOVR EPIC visible and NIR channels using MODIS Terra and Aqua data and EPIC lunar observations. Atmospheric Measurement Techniques 11, 359-368. DOI: 10.5194/amt-11-359-2018. 
8 Ohtake M, Matsunaga T, Yokota Y, Yamamoto S, Ogawa Y, Morota T, Honda C, Haruyama J, Kitazato K, Takeda H, Iwasaki A, Nakamura R, Hiroi T, Kodama S, and Otake H (2010). Deriving the absolute reflectance of lunar surface using SELENE (Kaguya) Multiband Imager data. Space Science Reviews 154, 57-77.

9 Ohtake M, Pieters CM, Isaacson P, Besse S, Yokota Y, Matsunaga T, Boardman J, Yamomoto S, Haruyama J, Staid M, Mall U, and Green RO (2013). One Moon, many measurements 3: Spectral reflectance. Icarus 226, 364-374.

$10 \mathrm{Su}$ W, Corbett J, Eitzen Z, and Liang L (2015). Next-generation angular distribution models for top-of-atmosphere radiative flux calculation from CERES instruments: methodology. Atmospheric Measurement Techniques 8, 611-632. DOI: 10.5194/amt-8-611-2015.

$11 \mathrm{Su} \mathrm{W}$, Corbett J, Eitzen Z, and Liang L (2015). Next-generation angular distribution models for top-of-atmosphere radiative flux calculation from CERES instruments: validation. Atmospheric Measurement Techniques 8, 3297-3313. DOI: 10.5194/amt-8-3297-2015.

12 Coddington O, Lean JL, Lindholm D, Pilewskie P, Snow M, and NOAA CDR Program (2015). NOAA Climate Data Record (CDR) of Solar Spectral Irradiance (SSI), NRLSSI Version 2. NOAA National Centers for Environmental Information. DOI:10.7289/V51J97P6, accessed 2020-10-21.

13 Goode PR, Qiu J, Yurchyshyn V, Hickey J, Chu M-C, Kolbe E, Brown CT, and Koonin SE (2001). Earthshine observations of the Earth's reflectance. Geophysical Research Letters, 28(9), 1671-1674. DOI: 10.1029/2000GL012580.

14 Hartmann D (2015). Global Physical Climatology. Elsevier Science. 2nd Edition, ISBN: 9780123285317, $498 \mathrm{pp}$.

15 ImageJ software. URL https://imagej.github.io/.

\section{Acknowledgements}

We gratefully acknowledge funding received from the Academy of Finland (grant numbers 298137 and 1333034). Computational resources are provided by CSC - IT Center for Science Ltd., Finland.

\section{Author contributions}

The method and algorithm were designed by A.P., K.M. O.I., E.U., M.V., G.X., and O.W.. The algorithm was coded by A.P., O.I., E.U., M.V., and O.W.. The web service was designed by A.P., K.M., E.U., M.V., and coded by A.P. and M.V.. The data in this article was prepared by A.P., as well as the statistical analysis on the data. The article was written by A.P., K.M., E.U., M.G., and H.J.. All authors provided input and comments to the article.

\section{Competing interests}

No 


\section{Materials \& Correspondence}

344 Antti Penttilä, antti.i.penttila@helsinki.fi 